\title{
Preserved miR-361-3p Expression Is an Independent Prognostic Indicator of Favorable Survival in Cervical Cancer
}

\author{
Shikai Liu, Lili Song $\mathbb{D}^{D}$, Hairong Yao, Liang Zhang, Dongkui Xu, Qian Li, and Ying Li \\ Department of Obstetrics \& Gynecology, Cangzhou Central Hospital, Hebei 061001, China \\ Correspondence should be addressed to Lili Song; lilisong11@126.com
}

Received 19 March 2018; Revised 9 July 2018; Accepted 26 July 2018; Published 23 September 2018

Academic Editor: Michele Malaguarnera

Copyright (c) 2018 Shikai Liu et al. This is an open access article distributed under the Creative Commons Attribution License, which permits unrestricted use, distribution, and reproduction in any medium, provided the original work is properly cited.

\begin{abstract}
In this study, we aimed to assess the independent prognostic value of miR-361-3p in terms of overall survival (OS) and recurrencefree survival (RFS) in cervical cancer, as well as its possible regulative network. A retrospective analysis was performed by using data from the Cancer Genome Atlas-Cervical Cancer (TCGA-CESC). Results showed that decreased miR-361-3p expression was associated with lymphovascular invasion and poor responses to primary therapy. The patients with recurrence and the deceased cases had substantially lower miR-361-3p expression compared to their respective controls. By generating Kaplan-Meier curves of OS and RFS, we found that high miR-361-3p expression was associated with better survival outcome. More importantly, univariate and multivariate analysis confirmed that high miR-361-3p expression was an independent indicator of favorable OS (HR: 0.377, 95\% CI: $0.233-0.608, p<0.001$ ) and RFS (HR: 0.398, 95\% CI: $0.192-0.825, p=0.013$ ). By performing bioinformatic analysis, we identified 24 genes that were negatively correlated with miR-361-3p expression. Among the potential targeting genes, SOST, MTA1, TFRC, and YAP1 are involved in some important signaling pathways modulating cervical cancer cell invasion, migration, and drug sensitivity. Therefore, it is meaningful to verify the potential regulative effect of miR-361-3p on the expression of these genes in the future.
\end{abstract}

\section{Introduction}

MicroRNAs (miRNAs) are a family of small, conserved, and noncoding RNAs that mainly exert regulative effect via repressing the transcription of targeting RNAs or inducing RNA degradation by binding to their $3^{\prime}$-UTR or $5^{\prime}$-UTR $[1,2]$. Homo sapiens miR-361 stem-loop encodes two miRNAs, including miR-361-5p and miR-361-3p. A series of studies found that miR-361-5p acts as a tumor suppressor in multiple cancers. It suppresses lung cancer progression by targeting FOXM1 [3]; inhibits hepatocellular carcinoma cell growth by targeting CXCR6 [4]; decreases glycolytic metabolism, proliferation, and invasion of breast cancer by targeting FGFR1 and MMP1 [5]; and prevents the malignant progression of prostate cancer cells by targeting STAT6 [6]. In comparison, the functional role of miR-361-3p in cancers was less studied. One recent study found that in nonsmall cell lung cancer (NSCLC), miR-361-3p could suppress tumor cell proliferation and metastasis by directly targeting SH2B1 [7].
Decreased miR-361-3p expression in prostate secretion samples might be an important diagnostic marker of prostate cancer [8].

Dysregulated miRNAs have been implicated in the pathological development of cervical cancer. Our previous studies found that miR-21 could modulate the radiosensitivity of cervical cancer cells through targeting LATS1 [9], while miR-375 could regulate the sensitivity by targeting UBE3A [10]. Some miRNAs have also been demonstrated as valuable prognostic markers in cervical cancer. For example, miR-155 upregulation was an independent prognostic indicator of overall survival for cervical cancer [11]. Downregulated serum level of miR-101 was an independent predictor of the unfavorable prognosis of cervical cancer [12].

However, the prognostic value of miR-361-3p in cervical cancer has not been explored. In this study, we assessed the independent prognostic value of miR-361-3p in terms of overall survival (OS) and recurrence-free survival (RFS) in cervical cancer, by using data from the Cancer Genome 
Atlas-Cervical Cancer (TCGA-CESC). In addition, we also examined the possible regulative network of miR-361-3p by performing a bioinformatic analysis.

\section{Materials and Methods}

2.1. Human Tissue Specimens. Cervical cancer tissues were obtained from 31 patients with primary cervical squamous cell carcinoma (SCC) who underwent radical trachelectomy or hysterectomy with pelvic lymph node dissection from July 2014 through December 2016 in Cangzhou Central Hospital. The mean age was 46.3 years (range, 31 to 68 years). Among the 31 cases, 23 cases were International Federation of Gynecology and Obstetrics (FIGO) stage I and 8 were stage II. All of the patients never received preoperative radiotherapy and/or chemotherapy before the tissues were collected. Normal cervical tissues with high-risk human papilloma virus (HR-HPV) infection $(N=19)$ or without HPV infection $(N=25)$ were obtained from patients who had trachelectomy or hysterectomy due to nonmalignant conditions. The tissue samples were immediately snapfrozen in liquid nitrogen and stored at $-80^{\circ} \mathrm{C}$ until RNA extraction. This study was approved by the Ethics Committee of Cangzhou Central Hospital.

2.2. QRT-PCR Analysis of miR-361-3p Expression. Total RNAs in tissue samples were extracted using the TRIzol reagent (Invitrogen, Carlsbad, CA, USA) according to manufacturer's instructions. miRNA specific cDNA was synthesized using the stem-loop primers ( $5^{\prime}$-GTC GTA TCC AGT GCA GGG TCC GAG GTA TTC GCA CTG GAT ACG AAA TCA GAA T- $3^{\prime}$ ) and the TaqMan microRNA reverse transcription kit (Applied Biosystems, Foster City, CA, USA). miR-361-3p expression was quantified by qRT-PCR, using TaqMan microRNA assays (Applied Biosystems). All PCR reactions were performed using an ABI Prism 7900 system (Applied Biosystems). The results were calculated using the $2^{-\Delta \Delta C T}$ methods.

2.3. Retrieving of Data in TCGA-CESC. The level-3 data in TCGA-CESC was obtained by using the UCSC Xena Browser (https://xenabrowser.net/). In this cohort, 308 patients with primary cervical cancer were included. Among these patients, 306 cases had miR-361-3p expression measured by RNAseq (IlluminaHiseq), 293 cases had intact OS data recorded, and 254 cases belong to squamous cell carcinoma (SCC). The clinicopathological data of the patients with intact OS data were downloaded for survival-related analysis, including age at initial pathologic diagnosis, histology, tumor grade, clinical stage, lymphovascular invasion indicator, primary therapy outcomes, the history of radiation therapy, targeted molecular therapy and postoperative drugs, recurrence status, and living status.

2.4. Bioinformatic Analysis of the Possible Targets of miR-361$3 p$ in Cervical Cancer. The possible targets of miR-361-3p were predicted using TargetScan 7.1, by setting the cumulative weighted context++ score $\leq-0.3$ as the threshold. The genes that were negatively correlated with miR-361-3p

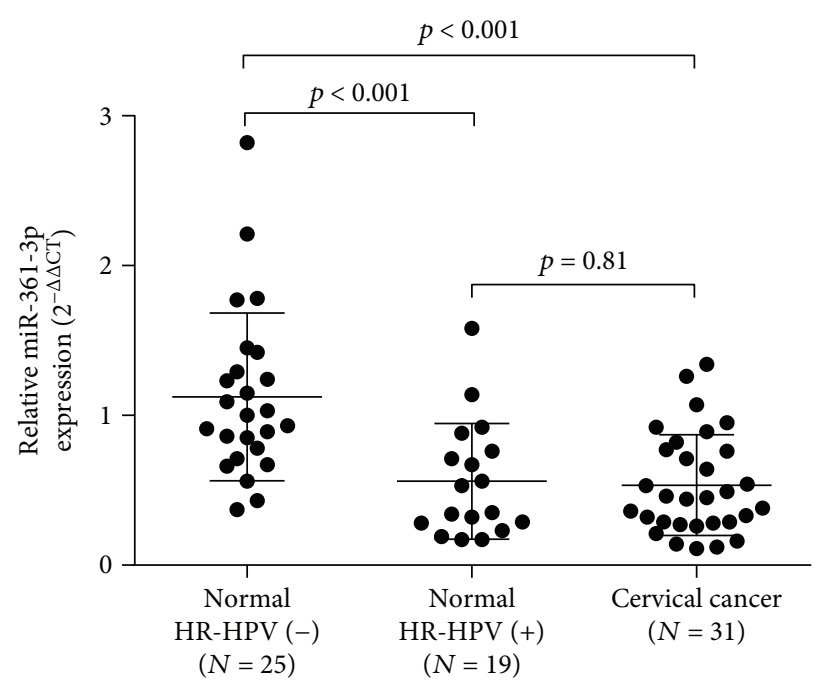

FIGURE 1: miR-361-3p expression profile. miR-361-3p expression was significantly decreased in $\mathrm{HR}-\mathrm{HPV}(+)$ normal cervical tissues $(N=19)$ and in cervical cancer tissues $(N=31)$ compared to that in HR-HPV $(-)$ normal cervical tissues $(N=25)$.

in TCGA-CESC were identified by setting Pearson's $r$ value $\leq-0.2$ as the threshold. The overlapping subset between the negatively correlated genes and the predicted target genes was identified. Then, the genes were loaded into ClueGO to analyze their functional annotations in Gene Ontology (GO). Only pathways with $p$ value $<0.05$ were included.

2.5. Statistical Analysis. Statistical analysis was performed by using GraphPad Prism 6.0 (GraphPad Inc., La Jolla, CA, USA) or SPSS 19.0 software package (SPSS Inc., Chicago, IL, USA). Welch's unequal variances $t$-test was conducted to compare the difference of $\mathrm{miR}-361-3 p$ expression in different groups. Receiver operating characteristic (ROC) analysis for death and recurrence detection was performed to identify the best cutoff (Youden index) for miR-361-3p expression in Kaplan-Meier curves. Log-rank test was applied to evaluate the significance of the difference between the survival curves. Fisher's exact test was used to assess the association between miR-361-3p expression and the clinicopathological parameters. Univariate and multivariate Cox regression analysis was conducted to determine the independent prognostic value of miR-361-3p expression in terms of OS and RFS. $p<0.05$ was considered statistically significant.

\section{Results}

3.1. Patients with Different Survival Outcomes Have Varying miR-361-3p Expressions. Using normal and tumor tissue samples, we found that the normal tissues with high-risk human papillomavirus (HR-HPV) infection $(N=19)$ had significantly decreased miR-361-3p expression compared with the normal tissues without the infection $(N=25)$ $(p<0.001)$ (Figure 1). However, we failed to identify 


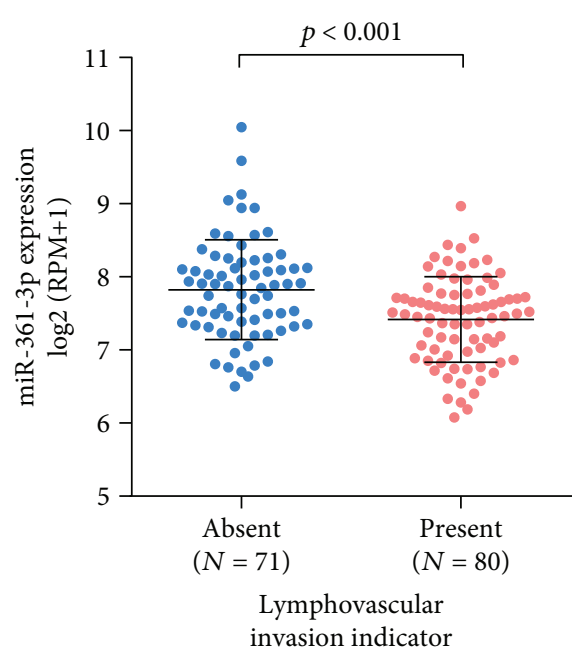

(a)

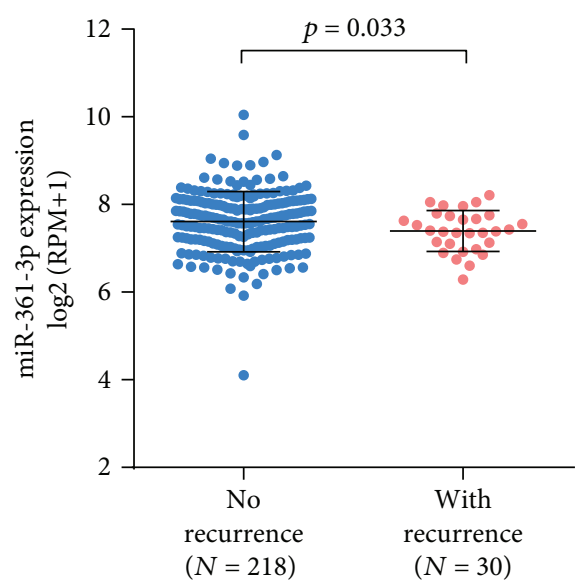

(c)

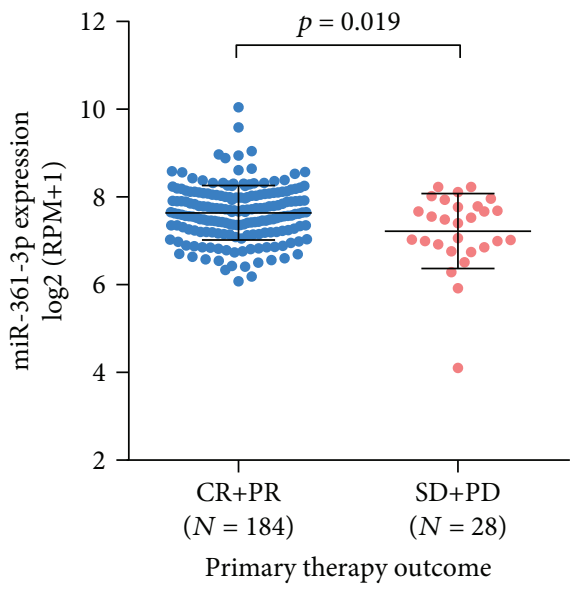

(b)

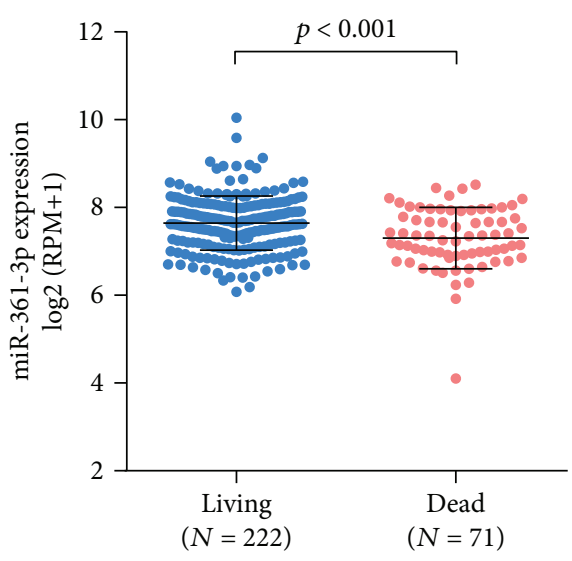

(d)

FIGURE 2: Decreased miR-361-3p was associated with malignant behaviors of cervical cancer. $(a, b)$ Comparison of miR-361-3p expression in cervical cancer patients with or without lymphovascular invasion (a) or in patients with response to primary therapy (CR and PR) or with no response to primary therapy (SD and PD) (b). (c, d) Comparison of miR-361-3p expression in cervical cancer patients with or without recurrence $(\mathrm{c})$ or in deceased or living patients $(\mathrm{d})$. CR: complete remission; PR: partial remission; SD: stable disease, and PD: progressive disease.

significant difference in miR-361-3p expression between HR-HPV (+) normal tissues and cancerous cervical cancer tissues (Figure 1). Using data from TCGA, we also compared miR-361-3p expression in 31 types of cancer with miRNA-seq data. Results showed that in colorectal cancer, thyroid cancer, and non-small-cell lung cancer, miR-361$3 \mathrm{p}$ expression tends to be downregulated in cancer tissues than in adjacent normal tissues (Supplementary Figure 1, red frames), while the opposite trend was observed in kidney clear cell carcinoma, stomach adenocarcinoma, bladder urothelial carcinoma, uterine corpus endometrioid carcinoma, and prostate adenocarcinoma (Supplementary Figure 1, green frames). These findings suggest the miR361-3p expression change might be cancer specific.

Then, we explored miR-361-3p expression between patients with different clinicopathological features. Results showed that the cases with lymphovascular invasion $(N=80)$ had significantly decreased miR-361-3p expression compared to the lymphovascular negative counterparts $(N=71, p<0.001)$ (Figure 2(a)). The patients with stable disease (SD) or progressive disease $(\mathrm{PD})(N=28)$ also had significantly lower miR-361-3p expression compared to the cases with complete remission (CR) or partial remission (PR) $(N=184)(p=0.019$, Figure 2(b)). However, no significant difference was observed between stage I/II and stage III/ IV diseases (Supplementary Figure 2). Then, we examined the discrepancy in miR-361-3p expression between the patients with different survival outcomes. Results showed that the cases with recurrence and the deceased cases had substantially lower miR-361-3p expression compared to their respective controls $(p=0.033$ and $p<0.001$, Figures 2 (c) and $2(\mathrm{~d})$ ).

3.2. Preserved miR-361-3p Expression Was an Independent Indicator of Favorable OS and RFS in Cervical Cancer. Since the patients with different survival outcomes had different 


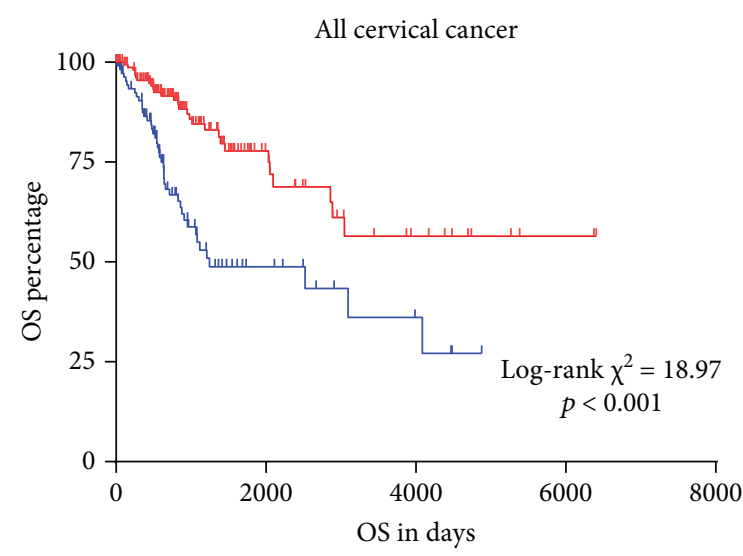

miR-361-3p expression

$+\operatorname{High}(N=176)$

$+\operatorname{Low}(N=117)$

(a)

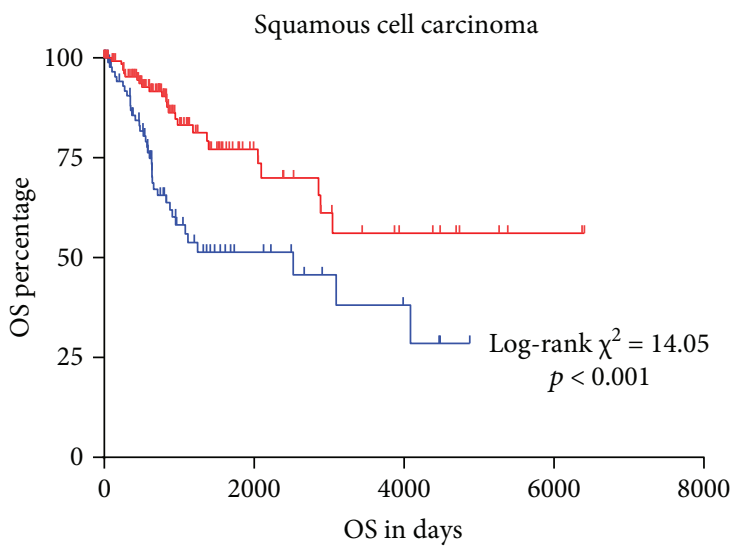

miR-361-3p expression

$+\operatorname{High}(N=146)$

$+\operatorname{Low}(N=97)$

(c)

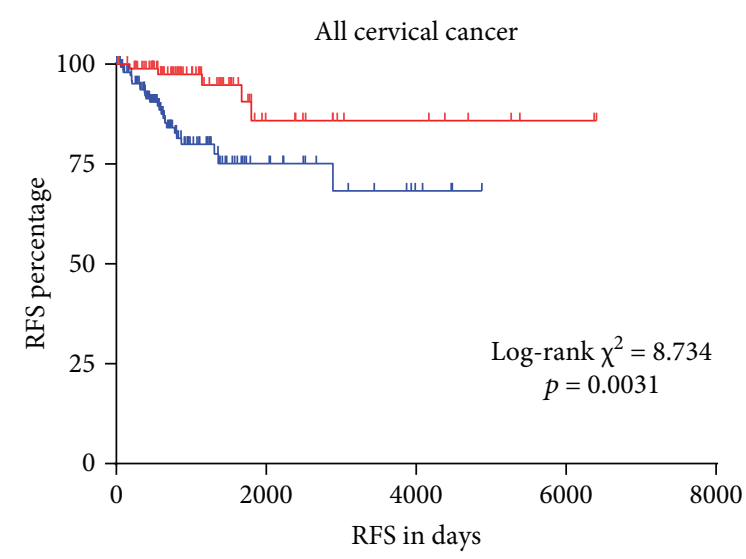

miR-361-3p expression

$+\operatorname{High}(N=95)$

$\perp \operatorname{Low}(N=153)$

(b)

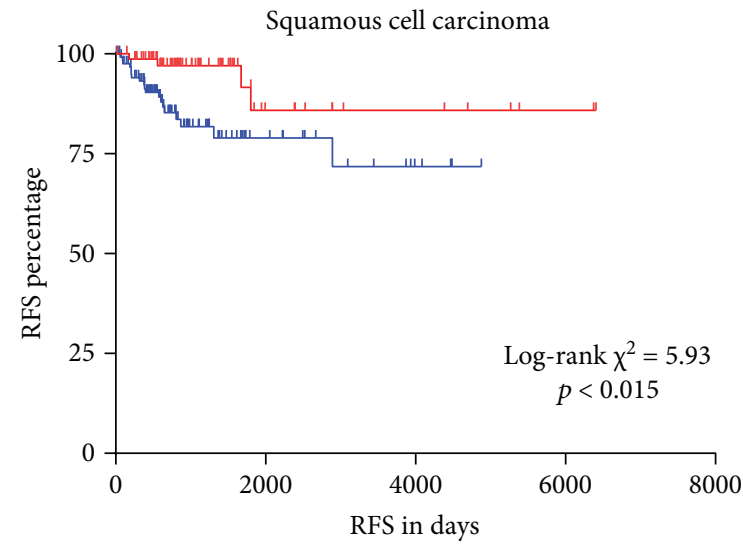

miR-361-3p expression

$\perp \operatorname{High}(N=78)$

$\perp \operatorname{Low}(N=126)$

FIGURE 3: Kaplan-Meier curves of OS and RFS in patients with cervical cancer. (a-d) Kaplan-Meier curves of OS (a, c) and RFS (b and d) in all cervical cancer patients $(a, b)$ and in patients with cervical squamous cell carcinoma $(c, d)$. Patients were grouped according to the optimal cutoff of miR-361-3p expression in the ROC curves for death and recurrence detection.

miR-361-3p expression, we decided to explore whether this miRNA is a prognostic marker in cervical cancer. By generating Kaplan-Meier curves of OS and RFS, we found that the low miR-361-3p expression group had significantly worse OS $(p<0.001)$ and RFS $(p=0.0031)$ compared to the high miR-361-3p expression group (Figures 3(a) and 3(b)). Since squamous cell carcinoma is the major subtype of cervical cancer, we also examined whether the associations were significant in this histological subtype. Using the same cutoff identified in Figures 3(a) and 3(b), we confirmed that low miR-361-3p expression was also significantly associated with unfavorable OS $(p<0.001)$ and RFS $(p=0.015)$ in squamous cell carcinoma (Figures 3(c) and 3(d)).

Then, we tried to investigate the independent prognostic value of $\mathrm{miR}-361-3 \mathrm{p}$ in cervical cancer. The associations between miR-361-3p expression and the clinicopathological parameters were summarized in Table 1. Results showed that the low miR-361-3p expression group had a significantly higher ratio of recurrence $(17 / 96$ vs. $13 / 152, p=0.044)$ and death $(43 / 117$ vs. $28 / 176, p<0.001)$ compared to the high miR-361-3p expression group (Table 1). No significant association was observed in other parameters, suggesting that these two groups were comparable. By performing univariate and multivariate analysis by using COX regression model, we found that early clinical stages (I/II), negative lymphovascular invasion, and high miR-361-3p expression (HR: $0.377,95 \%$ CI: $0.233-0.608, p<0.001)$ were independent indicators of favorable OS in cervical cancer (Table 2). By using RFS as the survival indicator, we also found that high miR-361-3p expression was an independent indicator of favorable RFS (HR: 0.398, 95\% CI: 0.192-0.825, $p=0.013$ ) in cervical cancer, after adjustment of lymphovascular invasion indicator (Table 3). 
TABLE 1: Comparison of the parameters between high and low miR-361-3p expression groups.

\begin{tabular}{|c|c|c|c|}
\hline \multirow[b]{2}{*}{ Parameters } & \multicolumn{2}{|c|}{ miR-361-3p expression } & \multirow[b]{2}{*}{$p$ value } \\
\hline & $\begin{array}{l}\text { High } \\
(N=176)\end{array}$ & $\begin{array}{c}\text { Low } \\
(N=117)\end{array}$ & \\
\hline Age $($ mean $\pm S D)$ & $47.91 \pm 13.32$ & $48.51 \pm 14.48$ & 0.72 \\
\hline \multicolumn{4}{|l|}{ Histology } \\
\hline $\begin{array}{l}\text { Squamous } \\
\text { cell carcinoma }\end{array}$ & 142 & 101 & \multirow{3}{*}{0.082} \\
\hline Adenocarcinoma & 32 & 12 & \\
\hline $\begin{array}{l}\text { Adenosquamous } \\
\text { carcinoma }\end{array}$ & 2 & 4 & \\
\hline \multicolumn{4}{|l|}{ Grade } \\
\hline G1/G2 & 90 & 57 & \multirow{3}{*}{0.71} \\
\hline G3/G4 & 70 & 49 & \\
\hline No data & 16 & 11 & \\
\hline \multicolumn{4}{|l|}{ Clinical stage } \\
\hline $\mathrm{I} / \mathrm{II}$ & 135 & 90 & \multirow{3}{*}{0.88} \\
\hline III/IV & 38 & 24 & \\
\hline No data & 3 & 3 & \\
\hline \multicolumn{4}{|l|}{$\begin{array}{l}\text { Lymphovascular } \\
\text { invasion indicator }\end{array}$} \\
\hline Absent & 49 & 21 & \multirow{3}{*}{0.093} \\
\hline Present & 45 & 35 & \\
\hline No data & 82 & 61 & \\
\hline \multicolumn{4}{|l|}{ Radiation therapy } \\
\hline No & 45 & 32 & \multirow{3}{*}{0.89} \\
\hline Yes & 108 & 73 & \\
\hline Null & 23 & 12 & \\
\hline \multicolumn{4}{|c|}{ Targeted molecular therapy } \\
\hline No & 38 & 29 & \multirow{3}{*}{0.36} \\
\hline Yes & 84 & 48 & \\
\hline No data & 54 & 40 & \\
\hline \multicolumn{4}{|l|}{ Postoperative drugs } \\
\hline No & 56 & 34 & \multirow{3}{*}{0.89} \\
\hline Yes & 81 & 46 & \\
\hline No data & 39 & 37 & \\
\hline \multicolumn{4}{|l|}{ Recurrence status } \\
\hline No & 139 & 79 & \multirow{3}{*}{0.044} \\
\hline Yes & 13 & 17 & \\
\hline Null & 24 & 21 & \\
\hline \multicolumn{4}{|l|}{ Living status } \\
\hline Living & 148 & 74 & \multirow{2}{*}{$<0.001$} \\
\hline Dead & 28 & 43 & \\
\hline
\end{tabular}

G1: well differentiated (low grade); G2: moderately differentiated (intermediate grade); G3: poorly differentiated (high grade); G4: undifferentiated (high grade).

3.3. Bioinformatic Analysis of the Possible Regulative Network of miR-361-3p in Cervical Cancer. Since one miRNA can participate in the regulation of multiple signaling pathways via targeting different mRNAs, we then explored the potential regulative network of miR-361-3p in cervical cancer.
Using online prediction software, we identified 791 predicted target genes of miR-361-3p, by setting cumulative weighted context++ score $\leq-0.3$ as the threshold (Figure 4). Then, we examined their coexpression with miR-361-3p in cervical cancer via their Pearson's $r$ value. By setting Pearson's $r$ value $\leq-0.2$ as the threshold, we identified 24 genes that were negatively correlated with miR-361-3p expression (Figure 4). To explore the potential signaling pathways in which miR361-3p might be involved in, the 24 genes were subjected to the analysis of functional annotations in Gene Ontology (GO). Results showed that in biological process, SOST was involved in the negative regulation of Wnt signaling pathway, MTA1 was involved in the positive regulation of protein autoubiquitination, NIN was involved in centrosome-templated microtubule nucleation, and RASL1OB was involved in the regulation of systemic arterial blood pressure by atrial natriuretic peptide (Figure 5). In molecular function, TFRC was involved in transferrin transmembrane transporter activity, while SLC6A2 was involved in norepinephrine:sodium symporter activity (Figure 5). In cellular component, YAP1 was involved in TEAD-1-YAP complex (Figure 5).

\section{Discussion}

There have been very limited studies that reported the regulative effect of miR-361-3p in cancers. One previous study found that miR-361-3p might act as a tumor suppressor in NSCLC and its antioncogenic activity may be due to its inhibition of the target gene SH2B1 [7]. miR-361-3p downregulation may promote lymph node metastasis of T1-stage colorectal cancer via the upregulation of E2F1 or $R A P 2 B$ expression [13]. In this study, we observed significantly lower miR-361-3p expression in HR-HPV-positive cases than in the negative cases. By analyzing the association between miR-361-3p expression and the clinicopathological parameters of cervical cancer, we found that preserved miR-361-3p expression was associated with better primary therapy responses and a lower ratio of lymphovascular invasion, recurrence, and death. However, no significant difference was observed between stage I/II and stage III/IV diseases, in terms of miR-361-3p expression. Therefore, we hypothesized that miR-361-3p downregulation might be related to HR-HPV infection. Its downregulation might consistently involve malignant transformation, progression, and therapeutic responses of cervical cancer. However, future molecular studies are required to verify these hypotheses. By generating Kaplan-Meier curves of OS and RFS, we found that high miR-361-3p expression was associated with better survival outcome. More importantly, univariate and multivariate analysis confirmed that high miR-361-3p expression was an independent indicator of favorable OS (HR: 0.377, 95\% CI: $0.233-0.608, p<0.001$ ) and RFS (HR: $0.398,95 \%$ CI: $0.192-0.825, p=0.013)$. These findings suggest that miR-361-3p might be a valuable prognostic biomarker of cervical cancer.

One miRNA might be involved in the regulation of multiple signaling pathways via targeting different mRNAs, and the targeting mRNAs might be cancer specific. To guide further exploration of the possible targets of miR-361-3p in 
TABLE 2: Univariate and multivariate analysis of OS in patients with cervical cancer.

\begin{tabular}{|c|c|c|c|c|c|c|c|c|}
\hline \multirow{3}{*}{$\begin{array}{l}\text { Parameters } \\
\text { Age }\end{array}$} & \multicolumn{4}{|c|}{ Univariate analysis } & \multicolumn{4}{|c|}{ Multivariate analysis } \\
\hline & \multirow{2}{*}{$\frac{p}{0.061}$} & \multirow{2}{*}{$\begin{array}{c}\text { HR } \\
1.017\end{array}$} & \multicolumn{2}{|c|}{$\begin{array}{c}95 \% \text { CI } \\
\text { (lower/upper) }\end{array}$} & \multirow[t]{2}{*}{$p$} & \multirow[t]{2}{*}{ HR } & \multicolumn{2}{|c|}{$\begin{array}{c}\text { 95\% CI } \\
\text { (lower/upper) }\end{array}$} \\
\hline & & & 0.999 & 1.035 & & & & \\
\hline \multicolumn{9}{|l|}{ Histology } \\
\hline Squamous cell carcinoma & & 1.000 & & & & & & \\
\hline Adenocarcinoma & 0.869 & 0.945 & 0.483 & 1.850 & & & & \\
\hline Adenosquamous carcinoma & 0.642 & 1.604 & 0.219 & 11.744 & & & & \\
\hline \multicolumn{9}{|l|}{ Clinical stage } \\
\hline III/IV vs. I/II & 0.001 & 2.248 & 1.373 & 3.679 & 0.066 & 1.610 & 0.970 & 2.674 \\
\hline \multicolumn{9}{|c|}{ Lymphovascular invasion indicator } \\
\hline Absent vs. present & 0.002 & 0.098 & 0.023 & 0.415 & 0.002 & 0.105 & 0.025 & 0.449 \\
\hline \multicolumn{9}{|l|}{ Radiation therapy } \\
\hline No vs. yes & 0.237 & 0.700 & 0.388 & 1.264 & & & & \\
\hline \multicolumn{9}{|l|}{ Targeted molecular therapy } \\
\hline No vs. yes & 0.701 & 1.114 & 0.643 & 1.930 & & & & \\
\hline \multicolumn{9}{|l|}{ Postoperative drugs } \\
\hline No vs. yes & 0.384 & 0.765 & 0.419 & 1.397 & & & & \\
\hline \multicolumn{9}{|l|}{ miR-361-3p expression } \\
\hline High vs. low & $<0.001$ & 0.362 & 0.225 & 0.583 & $<0.001$ & 0.377 & 0.233 & 0.608 \\
\hline
\end{tabular}

TABLE 3: Univariate and multivariate analysis of RFS in patients with cervical cancer.

\begin{tabular}{|c|c|c|c|c|c|c|c|c|}
\hline \multirow{3}{*}{$\begin{array}{l}\text { Parameters } \\
\text { Age }\end{array}$} & \multicolumn{4}{|c|}{ Univariate analysis } & \multicolumn{4}{|c|}{ Multivariate analysis } \\
\hline & \multirow{2}{*}{$\frac{p}{0.733}$} & \multirow{2}{*}{$\frac{\mathrm{HR}}{1.005}$} & \multicolumn{2}{|c|}{$\begin{array}{c}95 \% \text { CI } \\
\text { (lower/upper) }\end{array}$} & \multirow[t]{2}{*}{$p$} & \multirow[t]{2}{*}{ HR } & \multicolumn{2}{|c|}{$\begin{array}{c}\text { 95\% CI } \\
\text { (lower/upper) }\end{array}$} \\
\hline & & & 0.978 & 1.033 & & & & \\
\hline \multicolumn{9}{|l|}{ Histology } \\
\hline Squamous cell carcinoma & & 1.000 & & & & & & \\
\hline Adenocarcinoma & 0.426 & 1.441 & 0.586 & 3.545 & & & & \\
\hline Adenosquamous carcinoma & 0.222 & 3.535 & 0.466 & 26.821 & & & & \\
\hline \multicolumn{9}{|l|}{ Clinical stage } \\
\hline III/IV vs. I/II & 0.974 & 1.015 & 0.415 & 2.486 & & & & \\
\hline \multicolumn{9}{|c|}{ Lymphovascular invasion indicator } \\
\hline Absent vs. present & 0.006 & 0.128 & 0.029 & 0.562 & 0.007 & 0.131 & 0.030 & 0.572 \\
\hline \multicolumn{9}{|l|}{ Radiation therapy } \\
\hline No vs. yes & 0.399 & 0.679 & 0.276 & 1.670 & & & & \\
\hline \multicolumn{9}{|l|}{ Targeted molecular therapy } \\
\hline No vs. yes & 0.820 & 1.098 & 0.489 & 2.467 & & & & \\
\hline \multicolumn{9}{|l|}{ Postoperative drugs } \\
\hline No vs. yes & 0.499 & 0.694 & 0.241 & 2.000 & & & & \\
\hline \multicolumn{9}{|l|}{ miR-361-3p expression } \\
\hline High vs. low & 0.025 & 0.578 & 0.358 & 0.932 & 0.013 & 0.398 & 0.192 & 0.825 \\
\hline
\end{tabular}

cervical cancer, we performed bioinformatic analysis to identify the highly possible targets. Our results found 24 predicted genes that were negatively correlated with miR361-3p expression in cervical cancer. Among the genes, SOST is involved in the negative regulation of the Wnt signaling pathway, which is a critical signaling pathway modulating cervical cancer cell proliferation, migration, and angiogenesis $[14,15]$. MTA1 is involved in the positive regulation of protein autoubiquitination. In fact, this gene has been characterized as an important oncogene facilitating cervical cancer progression and metastasis $[16,17]$. Knockdown of MTA1 partly restores p53 and E-cadherin expression in cervical cancer cells and subsequently decreases their invasion, migration, and adhesion capabilities [18]. 


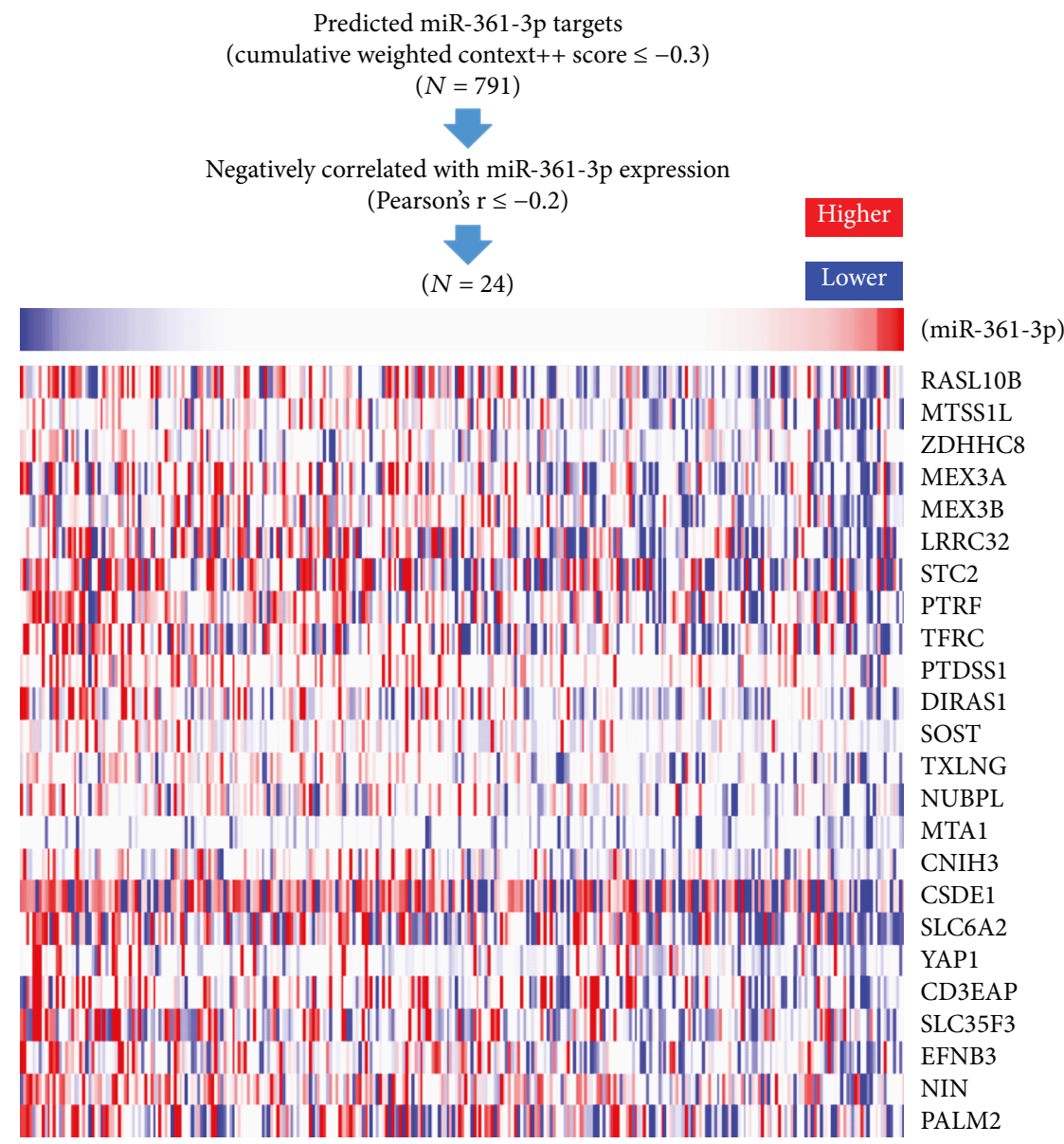

FIGURE 4: Screening of the genes negatively correlated with miR-361-3p in cervical cancer. Screening process showing the potential miR-361-3p targeting genes with negative correlation with miR-361-3p expression in cervical cancer tissues $(N=306)$.
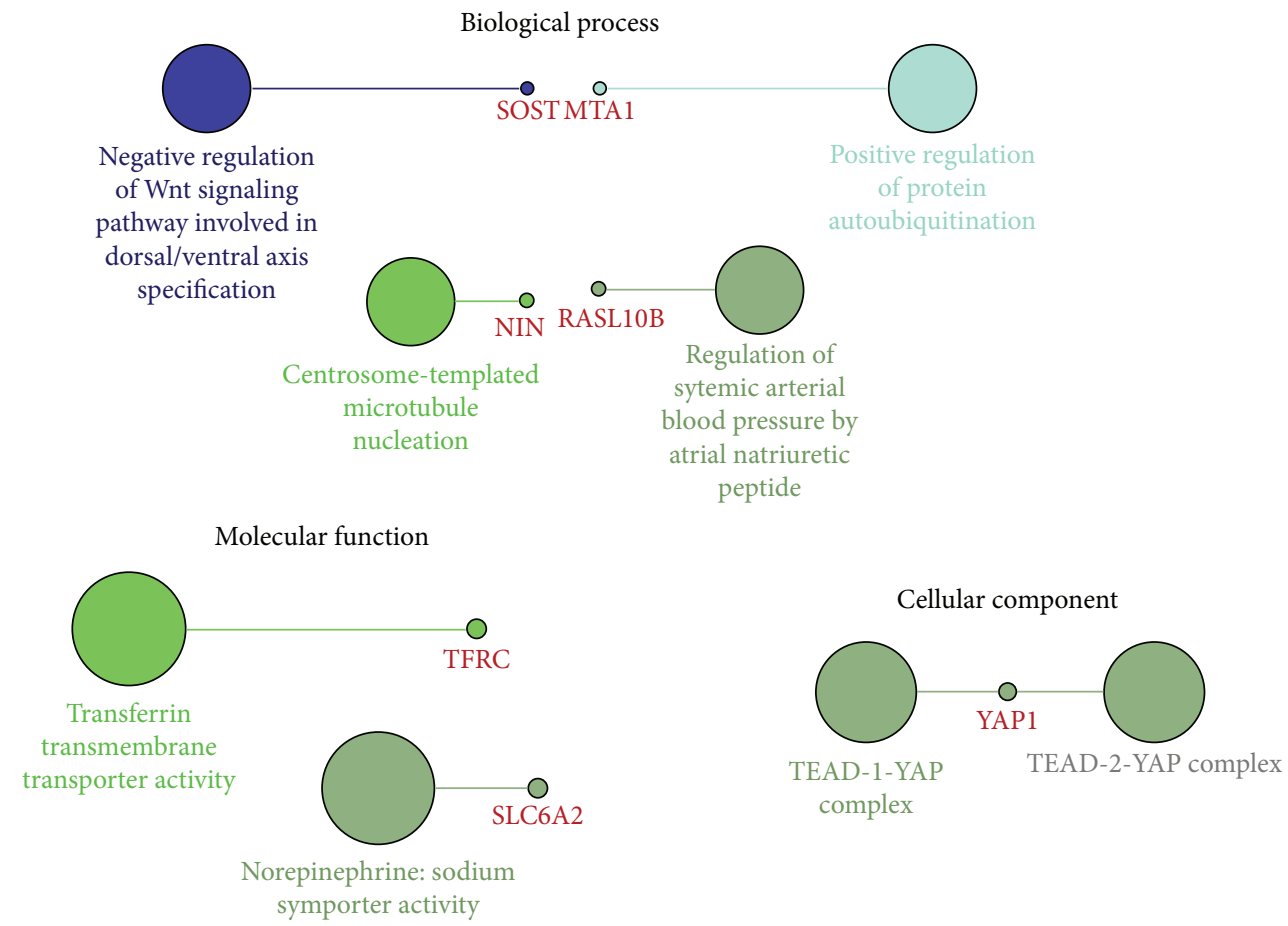

FIgURE 5: Functional annotations of the possible targets of miR-361-3p in cervical cancer in Gene Ontology. 
TFRC is significantly upregulated in cervical cancer and is considered as a candidate gene associated with the invasion of cervical cancer [19]. As an important component of the TEAD-1-YAP complex, YAP1 upregulation in cytoplasm is associated with histologic grading, lymph node metastasis, and recurrence of cervical SCC [20], and its nuclear overexpression is associated with shorter OS and disease-free survival of cervical adenocarcinoma [20]. YAP1 inhibition is associated with upregulation of p21 and p27 cell-cycle inhibitors and results in decreased cell proliferation, cell migration, anchorage-independent growth, and in vivo tumorigenic potential [21]. Besides, YAP1 silencing also increases the sensitivity of cervical cancer cells to cisplatin treatment [21]. Mechanistically, YAP expression is correlated with human papillomavirus (HPV) integration status and is required for the upregulation of TGF-alpha, amphiregulin (AREG), and EGFR, thereby forming a positive signaling loop to drive cervical cancer cell proliferation [22, 23]. In this study, we also found that miR-361-3p might be involved in the regulation of cervical cancer invasion and primary therapy responses. Therefore, it is meaningful to verify the potential regulative effect of miR-361-3p on the expression of these genes in the future.

\section{Conclusion}

Preserved miR-361-3p expression is an independent prognostic indicator of favorable OS and RFS in cervical cancer. It is meaningful to verify the potential regulative effect of miR-361-3p on the expression of SOST, MTA1, TFRC, and YAP1 in the future.

\section{Data Availability}

All relevant data were included in this article. The level-3 data in TCGA-OV used in this study can be accessed via the UCSC Xena Browser (https://xenabrowser.net/).

\section{Conflicts of Interest}

The authors have no conflict of interest.

\section{Supplementary Materials}

Supplementary Figure 1: the expression profile of miR-361$3 \mathrm{p}$ in tumors and adjacent normal tissues in TCGA. Supplementary Figure 2: miR-361-3p expression between stage I/II and stage III/IV cervical cancer. (Supplementary Materials)

\section{References}

[1] D. P. Bartel, "MicroRNAs: genomics, biogenesis, mechanism, and function," Cell, vol. 116, no. 2, pp. 281-297, 2004.

[2] V. Ambros, "The functions of animal microRNAs," Nature, vol. 431, no. 7006, pp. 350-355, 2004.

[3] X. W. Hou, X. Sun, Y. Yu et al., "miR-361-5p suppresses lung cancer cell lines progression by targeting FOXM1," Neoplasma, vol. 64, no. 4, pp. 526-534, 2017.

[4] J. J. Sun, G. Y. Chen, and Z. T. Xie, "MicroRNA-361-5p inhibits cancer cell growth by targeting CXCR6 in hepatocellular carcinoma," Cellular Physiology and Biochemistry, vol. 38, no. 2, pp. 777-785, 2016.

[5] F. Ma, L. Zhang, L. Ma, Y. Zhang, J. Zhang, and B. Guo, "MiR-361-5p inhibits glycolytic metabolism, proliferation and invasion of breast cancer by targeting FGFR1 and MMP1," Journal of Experimental \& Clinical Cancer Research, vol. 36, no. 1, p. 158, 2017.

[6] D. Liu, T. Tao, B. Xu et al., "MiR-361-5p acts as a tumor suppressor in prostate cancer by targeting signal transducer and activator of transcription-6(STAT6)," Biochemical and Biophysical Research Communications, vol. 445, no. 1, pp. 151-156, 2014.

[7] W. Chen, J. Wang, S. Liu et al., "MicroRNA-361-3p suppresses tumor cell proliferation and metastasis by directly targeting SH2B1 in NSCLC," Journal of Experimental \& Clinical Cancer Research, vol. 35, no. 1, p. 76, 2016.

[8] E. Guzel, O. F. Karatas, A. Semercioz et al., "Identification of microRNAs differentially expressed in prostatic secretions of patients with prostate cancer," International Journal of Cancer, vol. 136, no. 4, pp. 875-879, 2014.

[9] S. Liu, L. Song, L. Zhang, S. Zeng, and F. Gao, "miR-21 modulates resistance of HR-HPV positive cervical cancer cells to radiation through targeting LATS1," Biochemical and Biophysical Research Communications, vol. 459, no. 4, pp. 679-685, 2015.

[10] L. Song, S. Liu, S. Zeng, L. Zhang, and X. Li, "miR-375 modulates radiosensitivity of HR-HPV-positive cervical cancer cells by targeting UBE3A through the p53 pathway," Medical Science Monitor, vol. 21, pp. 2210-2217, 2015.

[11] H. Fang, D. Shuang, Z. Yi, H. Sheng, and Y. Liu, "Up-regulated microRNA-155 expression is associated with poor prognosis in cervical cancer patients," Biomedicine \& Pharmacotherapy, vol. 83, pp. 64-69, 2016.

[12] W. Jiang, J. J. Pan, Y. H. Deng, M. R. Liang, and L. H. Yao, "Down-regulated serum microRNA-101 is associated with aggressive progression and poor prognosis of cervical cancer," Journal of Gynecologic Oncology, vol. 28, no. 6, article e75, 2017.

[13] C. K. Jung, S. H. Jung, S. H. Yim et al., "Predictive microRNAs for lymph node metastasis in endoscopically resectable submucosal colorectal cancer," Oncotarget, vol. 7, no. 22, pp. 32902-32915, 2016.

[14] I. Ramachandran, E. Thavathiru, S. Ramalingam et al., "Wnt inhibitory factor 1 induces apoptosis and inhibits cervical cancer growth, invasion and angiogenesis in vivo," Oncogene, vol. 31, no. 22, pp. 2725-2737, 2011.

[15] X. Sun and Y. Liu, "Activation of the Wnt/ $\beta$-catenin signaling pathway may contribute to cervical cancer pathogenesis via upregulation of Twist," Oncology Letters, vol. 14, no. 4, pp. 4841-4844, 2017.

[16] T. Liu, M. Yang, S. Yang, T. Ge, L. Gu, and G. Lou, "Metastasisassociated protein 1 is a novel marker predicting survival and lymph nodes metastasis in cervical cancer," Human Pathology, vol. 44, no. 10, pp. 2275-2281, 2013.

[17] A. Bruning, T. Blankenstein, J. Juckstock, and I. Mylonas, "Function and regulation of MTA1 and MTA3 in malignancies of the female reproductive system," Cancer and Metastasis Reviews, vol. 33, no. 4, pp. 943-951, 2014.

[18] Y. Rao, H. Wang, L. Fan, and G. Chen, "Silencing MTA1 by RNAi reverses adhesion, migration and invasiveness of cervical cancer cells $(\mathrm{SiHa})$ via altered expression of $\mathrm{p} 53$, and 
E-cadherin/ $\beta$-catenin complex," Journal of Huazhong University of Science and Technology [Medical Sciences], vol. 31, no. 1, pp. 1-9, 2011.

[19] J. Y. Song, J. K. Lee, N. W. Lee, H. H. Jung, S. H. Kim, and K. W. Lee, "Microarray analysis of normal cervix, carcinoma in situ, and invasive cervical cancer: identification of candidate genes in pathogenesis of invasion in cervical cancer," International Journal of Gynecological Cancer, vol. 18, no. 5, pp. 10511059, 2008.

[20] T. Liu, Y. Liu, H. Gao, F. Meng, S. Yang, and G. Lou, "Clinical significance of yes-associated protein overexpression in cervical carcinoma: the differential effects based on histotypes," International Journal of Gynecological Cancer, vol. 23, no. 4, pp. 735-742, 2013.

[21] E. Lorenzetto, M. Brenca, M. Boeri et al., "YAP1 acts as oncogenic target of 11q22 amplification in multiple cancer subtypes," Oncotarget, vol. 5, no. 9, pp. 2608-2621, 2014.

[22] H. Xiao, L. Wu, H. Zheng et al., "Expression of yes-associated protein in cervical squamous epithelium lesions," International Journal of Gynecological Cancer, vol. 24, no. 9, pp. 1575-1582, 2014.

[23] C. He, D. Mao, G. Hua et al., "The Hippo/YAP pathway interacts with EGFR signaling and HPV oncoproteins to regulate cervical cancer progression," EMBO Molecular Medicine, vol. 7, no. 11, pp. 1426-1449, 2015. 


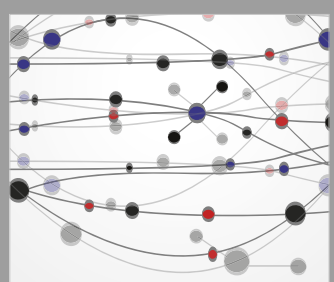

The Scientific World Journal
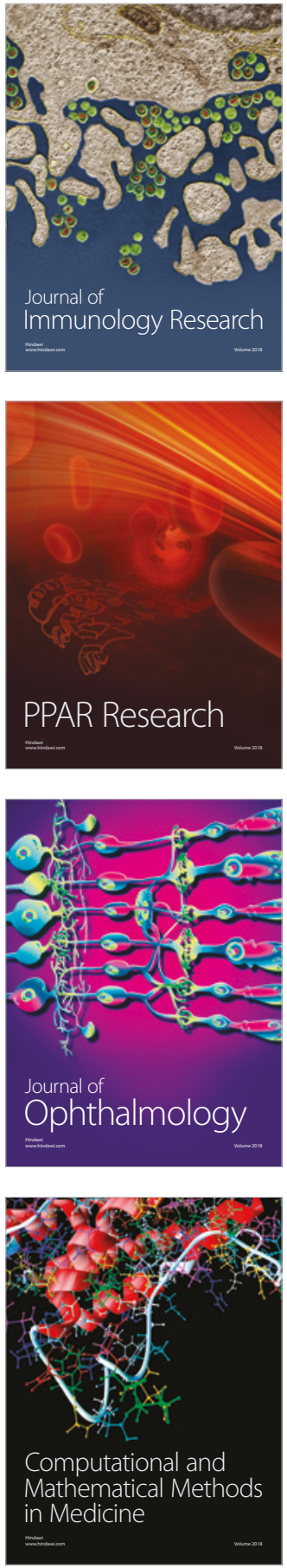

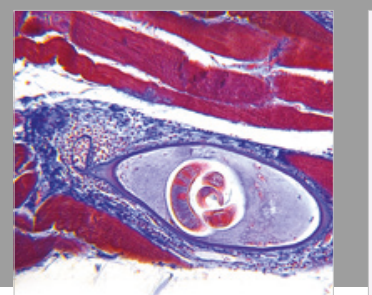

Gastroenterology Research and Practice

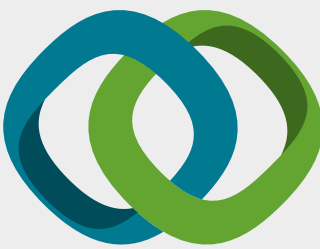

\section{Hindawi}

Submit your manuscripts at

www.hindawi.com
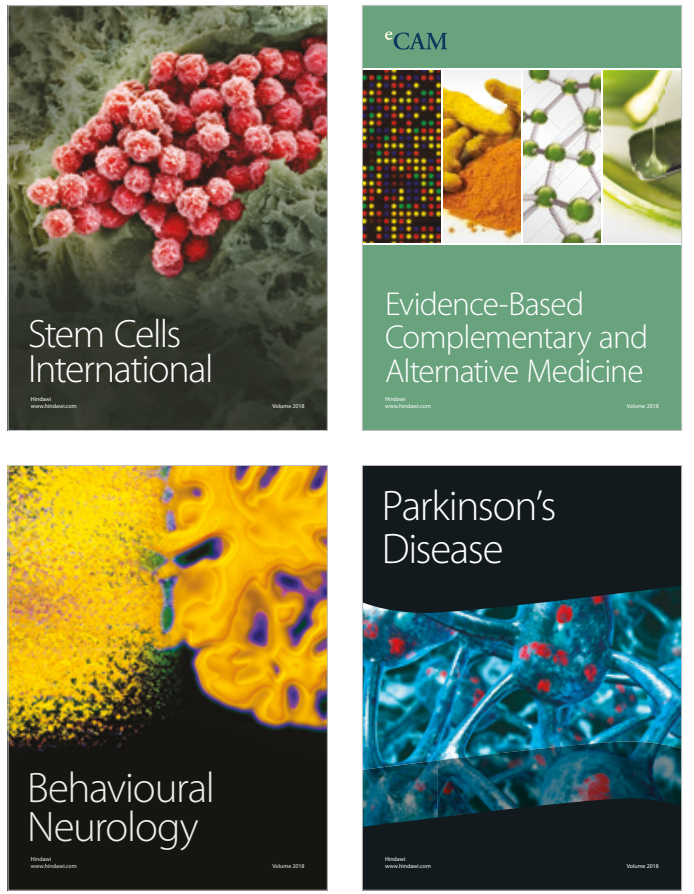

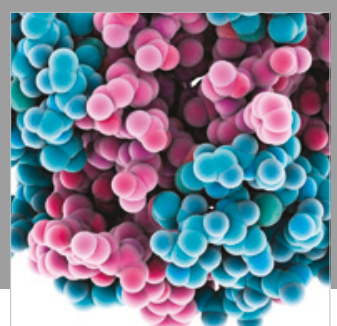

ournal of

Diabetes Research

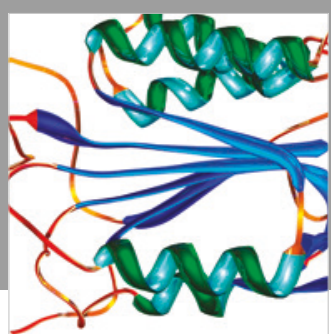

Disease Markers
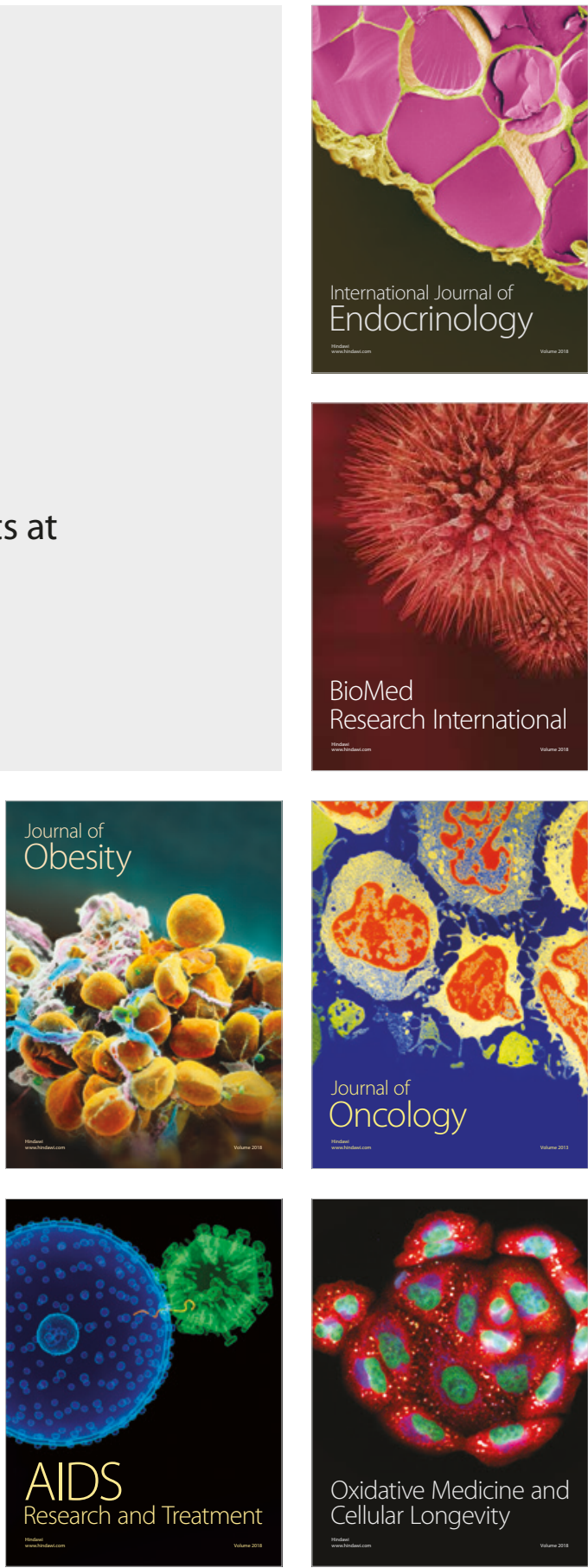